\title{
Fatal Basilar Arterial Thrombosis after Cardiac Surgery
}

\author{
Marzia Cottini, $\mathrm{MD}^{\mathrm{a}^{*}}$, Francesco Terrieri, $\mathrm{MD}^{\mathrm{b}}$, Gabriele Piffaretti, MD, $\mathbf{P h D}{ }^{\mathrm{b}}$, \\ Cesare Beghi, MD ${ }^{b}$ \\ ${ }^{a}$ Department of Heart and Vessels, Cardiac Surgery Unit and Heart Transplantation Center , "S. \\ Camillo-Forlanini" Rome, Italy \\ ${ }^{\mathrm{b}}$ Department of Surgical and Morphological Sciences, Varese University Hospital, University of \\ Insubria, Varese, Italy \\ *marzia.cottini@hotmail.it
}

\begin{abstract}
:
Objective: A correct valuation of risk factors and monitoring of the patient before and during cardiac procedure will reduce the incidence and the severity of central nervous system complications in particularly in emergency procedure. Cerebral arterial thrombosis is an uncommon presentation in cardiac surgery (frequency varies since $1.5 \%$ to $6 \%$ ). The number of emboli signals is significantly higher in the anterior circulation than the posterior. We reported a case of 62 year-old-man presenting a state of unconsciousness, tetra-paresis and facial paresis after cardiac surgery procedure. Computed-Tomography angiography revealed a big wormlike thrombus in the basilar artery and posterior cerebral arteries. There are no previous reports of total thrombosis of basilar artery after cardiac surgery.
\end{abstract}

Keywords: Thrombosis; Coagulation; Embolism; Cardiac Surgery; Basilar Artery.

Conflict of interest: None

Relationship with industry and financial disclosure: None

\section{INTRODUCTION}

Basilar arterial thrombosis is a rare event that is associated with high morbidity and mortality. The presence of the thrombus in the cerebral arteries causes an abrupt decrease of blood flow in the areas supplies by these vessels. $(1,2)$ Clinically silent infarction may be far more frequent and could contribute to long-term cognitive dysfunction in patients undergoing to cardiac surgery. There are no previous reports of total thrombosis of basilar artery and the posterior cerebral arteries after emergency cardiac surgery.

\section{CASE Report}

We reported a case of 62 year-old man presented for postero-lateral non-ST elevated myocardial infarction to our Institution. Cardiac Troponin I was $5.97 \mathrm{mg} / \mathrm{ml}$. A transthoracic echocardiogram documented a left ventricle with akinesia of the posterior wall, inferior basal septum and apical area, LVEDV 173 ml, LVESV 108 ml, and LVEF 37\%. A Conventional Coronary Angiography (CCA) has performed and revealed a critical coronary disease of the left anterior descending artery (LDA, 90\%), of the diagonal branch (DG, 60\%), of the left circumflex artery (LCA, 70\%) and the occlusion of the right coronary artery (RCA, 100\%). The patient progressively deteriorated versus a cardiogenic shock hence he underwent to surgery.

After the induction the patient was conscious and there wasn't neurological disorder.

The induction of the patient was done with Propofol $(1.4 \mathrm{mg} / \mathrm{Kg})$, Midazolan $(0.02 \mathrm{ml} / \mathrm{Kg})$, Suxamethonium Chloride $(1.5 \mathrm{mg} / \mathrm{Kg})$ and Rocuronium $(1 \mathrm{mg} / \mathrm{Kg})$. Then he started the infusion of Propofol (20 ml/h) and RemiFentanyl $(5 \mathrm{ml} / \mathrm{h})$.

The cardiac surgical procedure was carried out by central cardiopulmonary bypass (CPB) and it was performed three coronary artery bypass grafts (one by the left internal thoracic artery and two by left saphenous vein) without any operative complications. During the CPB time, the Activated Clotting 
Time (ACT) was in the range (more than $480 \mathrm{~s}$, heparin dose of $500 \mathrm{U} / \mathrm{Kg}$ ) and it was checked every 30 minutes during the CPB. He came off cardiopulmonary bypass in sinus rhythm with inotropic support (Levosimendan 0.1 gamma/Kg/min and Norepinephrine $0.05 \mathrm{gamma} / \mathrm{Kg} / \mathrm{min}$ ) and mechanical support (intra-aortic balloon pump). The cardiac index was $2.01 \mathrm{~min}^{-1} \mathrm{~m}^{2}$. After weaning from $\mathrm{CPB}$, the protamine $(2 \mathrm{mg} / \mathrm{Kg})$ was given for each $100 \mathrm{U}$ of injected heparin in the initial bolus dose. Neutralization of heparin was confirmed by ACT control (155 s). The patient was transferred to intensive care unit and he was monitoring accurately. After 24 hours, the patient was in a state of unconsciousness and had seizures. A neurological exam was performed: comatose level of consciousness, closed eyes, un-arousal, signs of diffuse cortical dysfunction (hypoxia, hypoglycemia, hyponatremia, hypotension), weak neurological reflexes and GCS 3. A brain computed tomography (bCT) revealed thrombosis of basilar artery and posterior cerebral arteries with flattening of subarachnoid sulcus and volume effect which hidden fourth ventricle (Figure $1 \mathrm{~A}, 1 \mathrm{~B}, 1 \mathrm{C}$ ). The EEG was isoelectric (Figure 2). The spontaneous INR at the second day was 1.34 with an activated partial thromboplastin time (APTT) 33.7, the direct and indirect Coombs' testes were negative He was treated with acetylsalicylic acid (ASA) and low molecular weight heparin (LMWH). The hemodynamic and metabolic states of the patient became worse and worse even if cardiac inotropic support (drugs and mechanical support). The patient died the 5th postoperative day. The autopsy documented an unknown atypical mass of the prostate. The prostatic adenocarcinoma was Gleason type G3 and TNM Classification type pT2 pN0 G2. The tumor could be the cause of the unusual thrombotic event.

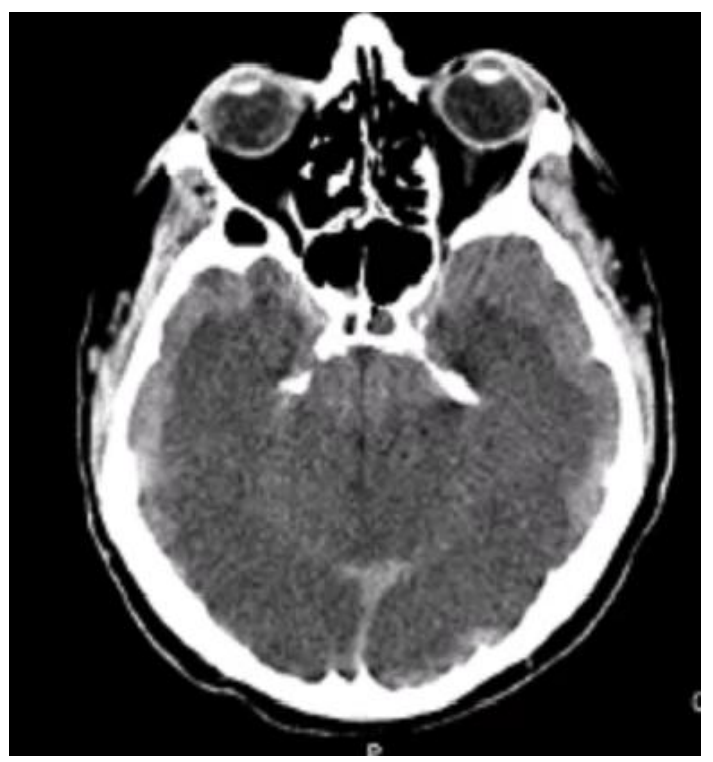

Figure1A. Computed Tomography of the Brain, Short-Axis View of the Hypodensity in the Posterior Cerebral Area

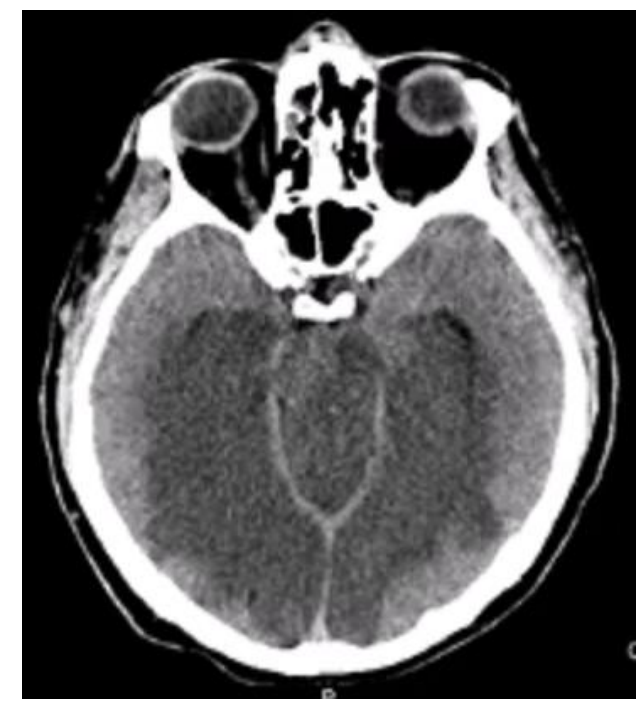

Figure1B. Computed Tomography of the Brain, Short-Axis View in Which is Evidence the Scraping of the Grooves of the Arachnoid and the Mass Effect Causing the Difficult Visualization of the Fourth Ventricle 


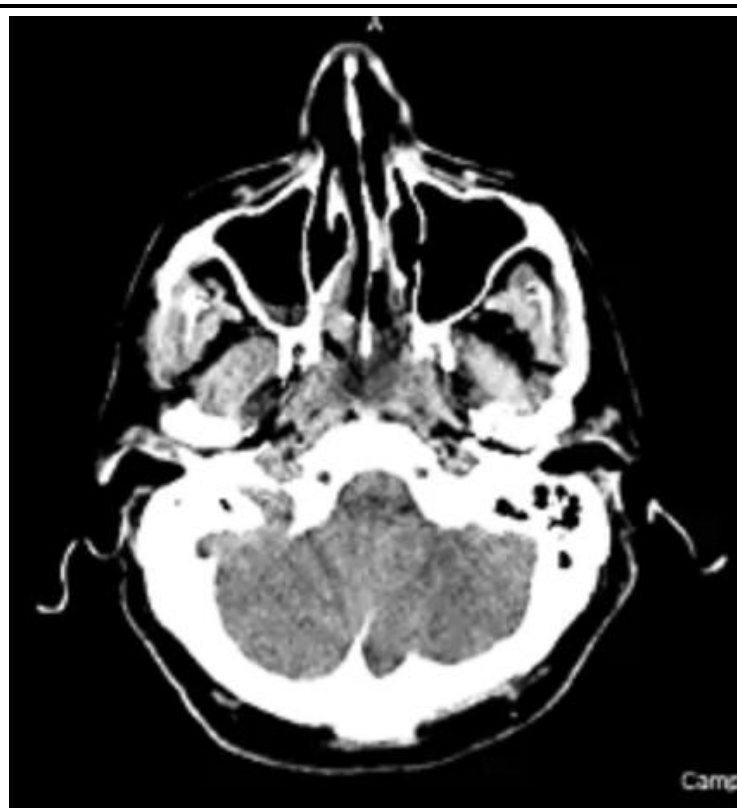

Figure1C. Computed Tomography of the Brain, Short-Axis View Documented the Free-Ischemic Cerebellar Area in Anterior and Inferior Side

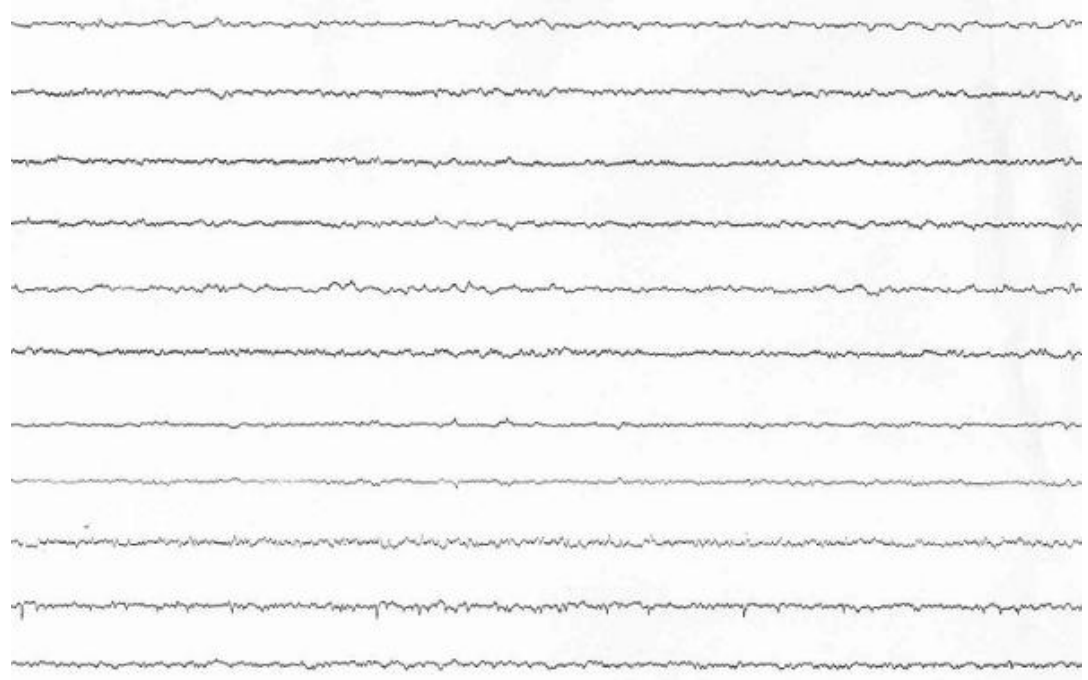

Figure2. The Isoelectric Electroencephalogram

\section{DisCuSSION}

Cerebral complications after cardiac surgery were widely studied. Advancing age increased the risk of stroke and cognitive impairment, especially after 6o year-old $(3,7)$.

The incidence of them depends on multiple factors, including age of patient, patient anamnesis, type of cardiac procedure, hypo perfusion or hyper perfusion during or after operation and so on (5) .

Methods to avoid the cerebral complications include: preoperative screening (when it is possible), intraoperative monitoring and postoperative observation (neurologic and neuropsychologist testing, electroencephalography and so on).

In case of emergency procedure, we don't know the anatomical situation of cerebral circulation. Therefore a specific and accurate monitoring of cerebral blood flow and metabolism measurement during cardiac surgery could crucial in preventing acute and long-term cerebral complications (4-8).

It is widely assumed that most brain injuries during adult cardiac surgery result from cerebral embolization of atheromatous or calcified material dislodged from sclerotic blood vessels during their manipulation. Technical developments have begun to alter this perception.

According to the literature, the brain is the most sensitive organ exposed to damage by CPB ad also the most important organ to protect. First, central nervous system (CNS) injuries still occur despite 
reductions in aortic manipulation with the new approaches to coronary artery bypass and aortic surgery. Second, neurophysiologic studies have implicated hypoperfusion and de-oxygenation as major causative factors in CNS injury. Because these functional disturbances are often detectable and correctable there is an impetus to examine the role of neurophysiologic monitoring in CNS protection. Multivariate (i.e., composed of several variables) descriptors have been developed to improve simple numeric characterization of clinically important EEG changes. With this approach, algorithms are used to generate a single number that represents the pattern of amplitude-frequency-phase relationships occurring in a single epoch. Several commercially available monitors provide unitless numbers that have been transformed to an arbitrary 0-to-100 scale. Current examples of these descriptors include the bispectral index (BIS), the patient state index (PSI), and spectral entropy (8-9).

BIS and PSI are empirically derived proprietary indices developed from proprietary patient databases. In contrast, spectral entropy is neither empirical nor proprietary but rather represents the novel application of long-established physical sciences entropy equations to the analysis of cranial biopotentials (10-12).

According to the sensibility of to detect the modification of cerebral blood flow, we divided the cerebral monitoring system in:

- Doppler system;

- Metabolic system;

- Neurophysiologic system.

The doppler system like neurophysiologic system tested the function of cerebral tissue, was non invasive, low-cost and suitable for operation room. One of the most important doppler system was trancranial doppler (TCD): it was important to evaluate the hemodynamic of cerebral blood flow that documented the cerebral maintenance of self-regulation and the vessel-reactivity by the trend of median velocity.

The metabolic system of cerebral monitoring included cerebral oxygenation measurement and it could be checked by invasive and non invasive procedure. The most popular procedure was the non invasive performing by an infrared light source located on the surface of two sticky patches that were placed on the scalp (Near Infrared Spectroscopy: NIRS).

The infrared light source transmitted photons through underlying tissues to the outer layers of the cerebral cortex, the NIRS measured all hemoglobin, not just the pulsatile arterial component contained in a mixed vascular bed primarily composed of gas-exchanging vessels, venules in particular. The measurement is thought to reflect $16 \%$ arterial and $84 \%$ venous contributions. This ratio remains nearly constant in normoxia, hypoxia, and hypocapnia and offered an objective measure of regional hypoperfusion (13-16).

The neurophysiologic cerebral monitoring system detected any modification in the electrical activity of the brain. They included electroencephalogram (EEG) and somatosensory evoked potential (SEP). The normal blood flow was about 50-80 $\mathrm{ml} / 100 \mathrm{~g} / \mathrm{min}$ and the mild cerebral hypoperfusion of 30 $\mathrm{ml} / 100 \mathrm{~g} / \mathrm{min}$ was well-tolerated and didn't cause modification in the synaptic communications. When the cerebral blood flow became less than $25 \mathrm{ml} / 100 \mathrm{~g} / \mathrm{min}$, the EEG and PES modified and documented cerebral electrical changes. The most characteristics signs in the EEG and PES monitoring were the reduction of amplitude, slow waves in the EEG and the increasing latency in the PES. Otherwise, EEG and PES had an important limitation: they disappeared when cerebral blood flow was less than $12-15 \mathrm{ml} / 100 \mathrm{~g} / \mathrm{min}$.

According to our experience, we suggested to use multimodality cerebral monitoring system such as EEG, NIRS and TCD. This combination elevated the quality and performance of cerebral monitoring system and with a complete detection of metabolic, neurophysiologic and blood flow features.

\section{CONClusion}

The current data of the best intraoperative cerebral monitoring were few and not enough to suggest a single high-quality system. In the cardiac surgery, the multimodal strategy could be the best cerebral monitoring to coordinate surgical procedure and anaesthesia, and to control the brain perfusion and metabolism in operation theatre. 


\section{REFERENCES}

[1] Thomas F. Floyd, Pallav N. Shah, Catherine C. Price, Francis Harris, Sarah J. Ratcliffe, Micheal A. Acker, Joseph E. Bavaria, Hind Rahmouni, Bettina kuersten, Susan Wiegers, Michael L. McGarvey, Joseph Y. Woo, Alberto A., Pochettino and Elias R. Melhem Clinically silent cerebral ischemic events after cardiac surgery: their incidence, regional vascular occurrence, and procedural dependence. Ann Thorac Surg 2006; 81: 2130-2166.

[2] Stephen A. Mills Cerebral Injury and cardiac operations. Ann Thorac Surg 1993; 56:S86-91.

[3] Hammon JW, Stump DA, Kon ND, et al. Risk factors and solutions for the development of neurobehavioral changes after coronary artery bypass grafting. Ann Thorac Surg 1998:63: 16131618 .

[4] Eric J. Heyer, Ellise Delphin, David C. Adams, Eric A. Rose, Craig R. Smith, George J. Todd, Mark Ginsburg, Rita Haggerty and Donald J. McMahon Cerebral dysfunction after cardiac operations in elderly patients. Ann Thorac Surg 1995; 60: 1716-1722.

[5] Fischer CM Basilar artery embolism after surgery under general anaesthesia: a case report. Neurology 1993 Sep; 43 (9): 1856-7.

[6] Hunaid A. Vohra, Amit Modi, Sunil K. Ohri. Does use of intraoperative cerebral regional oxygen saturation monitoring during cardiac surgery lead to improved clinical outcomes? Interactive Cardiovascular and thoracic Surgery, 2009, 9: 318-322.

[7] Stump DA, Brown WR, Moody DM et al. Microemboli and neurologic dysfunction after cardiovascular surgery. Semin Cardiothorac Vasc Anesth 1999, 3:47-54.

[8] Gugino L.D., Chabot R.J., Prichep L.S.D., et al: Quantitative EEG changes associated with loss and return of consciousness in healthy adult volunteers anaesthetized with propofol or sevoflurane. Br J Anaesth 2001; 87:421.

[9] Vakkuri A., Yli-Hankala A., Talja P., et al: Time-frequency balanced spectral entropy as a measure of anesthetic effect in central nervous system during sevoflurane, propofol, and thiopental anesthesia. Acta Anaesthesiol Scand 2004; 48:145.

[10] Viertiö-Oja H., Maja V., Särkelä M., et al: Description of the Entropy ${ }^{\mathrm{TM}}$ algorithm as applied in the Datex-Omeda S/5 ${ }^{\mathrm{TM}}$ Entropy Module. Acta Anaesthesiol Scand 2004; 48:154.

[11] Gugino L.D., Chabot R.J., Aglio L.S., et al: QEEG changes during cardiopulmonary bypass: Relationship to postoperative neuropsychological function. Clin Electroencephalogr 1999; 30:53.

[12] Newman M.F., Kirchner J.L., Phillips-Bute B., et al: Longitudinal assessment of neurocognitive function after coronary artery bypass surgery. N Engl J Med 2001; 344:395.

[13] Pigula F.A., Siewers R.D., Nemoto E.: Hypothermic cardiopulmonary bypass alters oxygen/glucose uptake in the pediatric brain. J Thorac Cardiovasc Surg 2001; 121:366.

[14] Monk T.G., Reno K.A., Olsen B.S., et al: Postoperative cognitive dysfunction is associated with cerebral oxygen desaturations. Anesthesiology 2000; 93:A167.

[15] Madsen P.L., Nielsen H.B., Christiansen P.: Well-being and cerebral oxygen saturation during acute heart failure in humans. Clin Physiol 2000; 20:158.

[16] Bar-Yosef S., Sanders E.G., Grocott H.P.: Asymmetric cerebral near-infrared oximetric measurements during cardiac surgery. J Cardiothorac Vasc Anesth 2003; 17:773. 